
\title{
3 Research Square \\ Role of salivary glycopatterns for oral microbiota associated with gastric cancer
}

\section{Jian Shu}

Northwest University https://orcid.org/0000-0002-2938-4218

Hanjie Yu

Northwest University

Xiameng Ren

Northwest University

Yan Wang

Northwest University

Kun Zhang

Northwest University

Zhen Tang

Northwest University

Liuyi Dang

Northwest University

Wentian Chen

Northwest University

Baozhen Li

The First Affiliated Hospital of Xi'an Jiaotong University

Zheng Li ( $\nabla$ zhengli@nwu.edu.cn )

Northwest University

\section{Research Article}

Keywords: gastric cancer, neoglycoproteins, fucosylated glycans, oral microbiota, A. segnis

Posted Date: December 9th, 2021

DOl: https://doi.org/10.21203/rs.3.rs-221122/v3

License: (9) This work is licensed under a Creative Commons Attribution 4.0 International License. Read Full License 


\section{Abstract}

Microbiota in the oral cavity plays an important role in maintaining human health. Our previous studies have revealed significant alterations of salivary glycopatterns in gastric cancer (GC) patients, but it is unclear whether these altered salivary glycopatterns can cause the dysbiosis of oral microbiota. In this study, the oral microbiome of healthy volunteers and GC patients were detected. The neoglycoproteins were then synthesized according to the altered glycopatterns in GC patients and used to explore the effects of specific glycopattern against oral microbiota. The results showed that the fucoseneoglycoproteins could reduce the adhesion and toxicity of $A$. segnis to oral cells, change the glycan structures of lipopolysaccharide, and enhance the immunogenicity of $A$. segnis. This study revealed that the changes of salivary glycopatterns in GC patients might contribute to the dysbiosis of oral microbiota, had important implications in developing new carbohydrate drugs to maintain a balanced microbiota in the oral.

\section{Introduction}

The human oral cavity is the natural port of entry to both the gastrointestinal and respiratory tracts, harbours more than 700 kinds of microbial species which interaction with host cells, microenvironment and each other, together to forms an equilibrium community normally (Escapa et al., 2018; Lamont, Koo, \& Hajishengallis, 2018). The balanced oral microbiome in symbiosis with the host is critical for the promotion and maintenance of oral and physical health. Increasing evidences have indicated that the oral microbiota is highly associated with human systemic diseases, including diabetes, obesity, and a wide variety of cancers (Belda-Ferre et al., 2012; X. Fan et al., 2018; Flemer et al., 2018; Long et al., 2017; Peters et al., 2017). The associations between oral microbiota and tumor are divided into two categories (Irfan, Delgado, \& Frias-Lopez, 2020). First, microorganisms are definitely involved in tumorigenesis, such as Fusobacterium nucleatum to colorectal cancer, Porphyromonas gingivalis to oral squamous cell carcinoma(Shang \& Liu, 2018; Whitmore \& Lamont, 2014). Several underlying mechanisms of microbiota induced-carcinogenesis, e.g. excessive inflammatory reaction, immunosuppression of host, promotion of malignant transformation, antiapoptotic activity, and secretion of carcinogens, have been proposed (Meurman, 2010; Sun et al., 2020; Whitmore \& Lamont, 2014). Second, oral microbiota is not directly involved in the pathogenesis of tumor, but consistent changes in the composition of oral microbiota appeared in tumor patients, which have the potential for tumor diagnosis (Farrell et al., 2012; Flemer et al., 2018). It remains controversial whether cancer is the cause or consequence of changes in the microbiota until now. Therefore, the relationship between oral microbiota and distant tumors still requires further elucidation.

Gastric cancer (GC) is a malignant tumor worldwide, which poses a serious threat to human health. The profiles of oral microbiota in GC patients have not been investigated, although some studies have focused on the tongue coating microbiota community of GC patients and the several specific oral pathogens in gastric precancerous lesions patients (Sun et al., 2017; Wu et al., 2018). Exploring the differences of oral microbiota in healthy volunteers (HV) and GC patients, and thoroughly understanding 
their relationship might provide a new insight into the development of non-invasive diagnosis, risk assessment, efficacy assessment of GC, and even the targeted treatment. Recent studies have shown that the special glycan and glycoprotein play a particularly important role in modulating the oral microbiota (Cross \& Ruhl, 2018; Lynge Pedersen \& Belstrøm, 2019). The glycans and glycoproteins on the host cell surface may foster and maintain microbiota adhesion and colonization by interacting with bacterial glycan receptors, and can be foraged as a microbial nutrient source degraded by a variety of glycoside hydrolases (Yang et al., 2016). On the contrary, the glycans attached to exogenous or secreted salivary glycoproteins may block or modulate pathogen association to the epithelial surface. It is well established that highly glycosylated proteins such as salivary mucins and proline-rich proteins have the capacity to bind and aggregate microorganisms whereby inhibiting their adhesion and planting (Lynge Pedersen \& Belstrøm, 2019). Furthermore, several specific glycan structures, such as a1,4GIcNAc in the scaffold proteins, can directly inhibit the growth of Helicobacter pylori (Kawakubo et al., 2004).

Our previous studies have demonstrated that the salivary glycopatterns were significantly altered in GC patients, including the decreased expression level of fucose expression profile, the increased expression level of mannose, GalNAc and galactose expression profiles (Shu et al., 2018; Shu, Yu, Li, Zhang, \& Liu, 2017). However, the association between salivary glycopatterns and oral microbiota in GC patients is unclear. In this study, the oral microbiota of HV and GC patients was firstly compared to screen the upregulated abundance bacteria in GC using $16 \mathrm{~S}$ rDNA sequencing. Then, the neoglycoproteins were synthesized according to the altered glycopatterns in GC patients and used to explore the effects of special salivary glycopattern against the increased bacteria. The purpose of this study is to investigate the differences of oral microbiota between HV and GC patients and to explore the relationship between salivary glycopatterns and oral microbiota, which could reveal the role of special glycan moieties and oral microbiota in the genesis and development of GC.

\section{Materials And Methods}

\section{Study Population and Sampling}

The collection of human salivary samples for the research presented here were approved by the Ethical Committee of Northwest University (Xi'an, China), First Affiliated Hospital of Xi'an Jiaotong University (Xi'an, China). Written informed consent was received from participants for the collection of their saliva samples. This study was conducted in accordance with the ethical guidelines of the Declaration of Helsinki. After a standardized endoscopic procedure and histopathological evaluation, individuals diagnosed with $\mathrm{GC}(\mathrm{N}=12)$ were enrolled in this study. Patients who received preoperative radiotherapy, chemotherapy, chemoradiotherapy or antibiotic therapy were excluded from the study. The enrolled HVs $(\mathrm{N}=12)$ were age/sex-matched and free of any precancerous lesions. No significant differences were observed between the two groups regarding demographic, socioeconomic, and lifestyle characteristics, and all the participants were $\geq 40$ years old. Sampling was performed in the morning before the 
participants had eaten breakfast. More than $1 \mathrm{~mL}$ unstimulated saliva samples were collected and immediately stored at $-20^{\circ} \mathrm{C}$. The clinical characteristics of participants are summarized in Table S1.

\section{Sequencing and Data Analysis}

Bacterial genomic DNA was extracted by a Bacterial DNA Isolation Kit D3350-01 (OMEGA, USA) following the manufacturer's instructions. The 16S rDNA V3-V4 regions were amplified by universal primers $341 \mathrm{~F}$ and 806R. Each forward primer containing a unique barcode (identification tag sequence) to distinguish samples. All PCR reactions were carried out with Phusion High-Fidelity PCR Master Mix (New England Biolabs, USA). The PCR products were extracted and quantified, pooled in equimolar concentrations and sequenced using the lon $\mathrm{S} 5^{\mathrm{TM}} \mathrm{XL}$ platform according to the manufacturer's recommendations.

Sequencing libraries were generated using Ion Plus Fragment Library Kit 48 rxns (Thermo Scientific, USA) following manufacturer's recommendations. The library quality was assessed on the Qubit 2.0 Fluorometer (Thermo Scientific, USA). At last, the library was sequenced on an lon S5TM XL platform, and $400 \mathrm{bp} / 600 \mathrm{bp}$ single-end reads were generated. Single-end reads was assigned to samples based on their unique barcode and truncated by cutting off the barcode and primer sequence. Quality filtering on the raw reads were performed under specific filtering conditions to obtain the high-quality clean reads according to the Cutadapt (http://cutadapt.readthedocs.io/en/stable/) quality-controlled process. The reads were compared with the reference database (Gold database,

http://drive5.com/uchime/uchime_download.html) to detect chimera sequences, and then the chimera sequences were removed. Then the effective sequences finally obtained and with $\geq 97 \%$ similarity were assigned to the same operational taxonomic units (OTUs). The representative sequence for each OTU was subject to BLASTN analysis against the HOMD database, Ribosomal Database Project and Greengenes for annotation (Dewhirst et al., 2010). OTUs abundance information were normalized and used for subsequent analysis. Then the analysis of alpha diversity and beta diversity were performed as described in previous reports (Ling et al., 2015). Differences between two groups were tested by the Wilcoxon test for each species using SPSS statistics 20.

\section{Synthesis of Neoglycoproteins}

The synthesis of neoglycoprotein is divided into three steps (Fig S1A): the monosaccharides coupled with the linker (5-(4-hydroxyphenyl)pentanoic acid) to form glycan conjugates, and the carboxylates (-COOH) from glycan conjugates reacted to N-hydroxysuccinimide (NHS) in the presence of 1-ethyl-3-(3dimethylaminopropyl (EDC), resulting in a semi-stable NHS, followed by reaction with amines (-NH2) of BSA to form amide crosslinks. Briefly, $5 \mu \mathrm{mol}$ of each monosaccharide (fucose, mannose, and galactose (Sigma-Aldrich, USA)) was respectively mixed with $2.5 \mu \mathrm{mol}$ of 5-(4-hydroxyphenyl) pentanoic acid, dissolved in $\mathrm{N}, \mathrm{N}$-dimethylformamide (DMF), decreased to a pH under 3.0 using sulfuric acid, and then incubated at $60^{\circ} \mathrm{C}$ for $12 \mathrm{~h}$ under gentle shaking. After chloroform extraction, the glycan complex was mixed with $10 \mu \mathrm{mol}$ EDC and $25 \mu \mathrm{mol}$ NHS in activation buffer (0.1 M MES (2-[morpholino] ethanesulfonic acid), $0.5 \mathrm{M} \mathrm{NaCl}, \mathrm{pH} 6.0$ ) and incubated at room temperature for $4 \mathrm{~h}$. Third, the products were added to $100 \mathrm{nmol}$ BSA solution (BSA dissolved in PBS), and then the buffer $\mathrm{pH}$ was adjusted to 7.4 
and incubated at room temperature for $4 \mathrm{~h}$. The quench reaction was performed by adding hydroxylamine to a final concentration of $10 \mathrm{mM}$. The synthesized neoglycoproteins were dialyzed against PBS overnight and concentrated using an Amicon ultra-4 $30 \mathrm{kDa}$ ultrafiltration unit (Millipore, USA). The neoglycoprotein solutions were collected, filtered through $0.22 \mu \mathrm{m}$ filters and stored at $-80^{\circ} \mathrm{C}$. The synthesized neoglycoproteins were analyzed by $10 \%$ SDS-PAGE and then stained directly with alkaline silver. Compared with the band of BSA, the upward migration bands of neoglycoproteins are obviously observed in the SDS-PAGE results (Fig S1B).

\section{Bacterial Strains and Cell Culture}

A. segnis was obtained from the American Type Culture Collection (ATCC) and cultured at $37^{\circ} \mathrm{C}$ with agitation (225 rpm) in tryptic soy broth (TSB) supplemented with $15 \mu \mathrm{g} / \mathrm{mL}$ NAD (Solarbio, China), goat serum (1:100 dilution) and $30 \mu \mathrm{g} / \mathrm{mL}$ vancomycin (Solarbio, China). CAL-27, HOEC and RAW264.7 cells were obtained from Otwo Biotech Inc. (Shenzhen, China), cultured in DMEM (HyClone, Waltham, MA, USA) supplemented with $10 \% \mathrm{FBS}$ (Gibco, Grand Island, NY, USA) and maintained at $37^{\circ} \mathrm{C}$ with $5 \% \mathrm{CO}_{2}$.

\section{RNA Extraction and qPCR}

Total RNA was extracted using the Bacteria Total RNA Isolation Kit (Sangon Biotech, China) and converted into cDNA using the PrimeScript RT reagent Kit (TaKaRa, Japan). The cDNAs were then subjected to real-time quantitative polymerase chain reaction (RT-qPCR) analysis ( $A B I$ ViiA ${ }^{T M} 7$, Bio-Rad, USA) using gene-specific primers (Table S2) and TB Green Fast qPCR Mix (TaKaRa, Japan). The 16S rRNA gene was used as an endogenous control for normalizing the relative expression of target genes.

\section{Adhesion Assays}

A. segnis was washed with fresh medium three times, centrifuged and diluted with fresh medium containing $100 \mu \mathrm{M}$ FITC-d-Lys (Xiamen Bioluminor Bio-Technology, China). After 30 min incubation at $37^{\circ} \mathrm{C}$, the cells were centrifuged, washed with fresh medium three times, and then resuspended in PBS. The CAL- 27 or HOEC cells $\left(1 \times 10^{5}\right.$ cells) were inoculated into confocal culture dishes (JingAn Biotechnology, China) and cultured in complete medium until the cells grown to $70-80 \%$ confluence, and then synchronized by serum starvation overnight. $100 \mu \mathrm{L}$ serum-free DMEM with $5 \mu \mathrm{M}$ DiD (AAT Bioquest, USA) were pipetted onto the dishes and gently agitate until all cells were covered. After 30 min incubation at $37^{\circ} \mathrm{C}$, the cells were washed with DMEM three times ( $10 \mathrm{~min}$ each) to remove the unlinked DiD. FITClabelled $A$. segnis $\left(1 \times 10^{6} \mathrm{cells} / \mathrm{mL}\right)$ was added into the dishes in $500 \mu \mathrm{L}$ serum-free DMEM medium and allowed to infect HOEC or CAL-27 for $45 \mathrm{~min}$ for adhesion. The infected cells were washed three times with PBS (5 min each), and immobilized by incubating with $0.2 \%$ Triton X-100 in $4 \%$ paraformaldehyde for 30 min at RT. After DAPI staining (Thermo Fisher Scientific), a laser scanning confocal microscope FV 1000 (Olympus, Tokyo, JPN) was used to acquire the images with the merged channels of FITC (Ex/Em $(\mathrm{nm})=488 / 520)$, DiD $(\operatorname{Ex} / \operatorname{Em}(\mathrm{nm})=650 / 670)$ and DAPI $(\operatorname{Ex} / \operatorname{Em}(n m)=358 / 461)$.

\section{Cell Viability Assay}


The cells were seeded in 96-well plates at a density of about 2000 cells per well. $0-3 \mu \mathrm{g} / \mathrm{mL}$ Fuc-BSA was added in the cells in order to assess the effect of Fuc-BSA to CAL-27 and HOEC cells. And the A. segnis (MOI 1, 5, 10, 20 and 50) and $100 \mu \mathrm{g} / \mathrm{mL}$ Fuc-BSA were added in the wells to test the influence of FucBSA in the infection of $A$. segnis to oral cells. The cell viability was assessed using CCK8 following manufacturers' instructions at $0,12,24,36,48$ and $72 \mathrm{~h}$.

\section{Lectin Microarrays and Data Analysis}

A lectin microarray was produced using 37 lectins with different binding preferences covering $\mathrm{N}$ - and $\mathrm{O}$ linked glycans(Shu et al., 2021). The Cy3-labelled glycoproteins or bacteria were incubated on the lectin microarray at $37^{\circ} \mathrm{C}$ for $3 \mathrm{~h}$ with gentle rotation in the dark. The slides were washed three times with PBST and PBS, respectively, centrifuged dry and scanned immediately with the $70 \%$ photomultiplier tube and $100 \%$ laser power settings using a Genepix 4000B confocal scanner (Axon Instruments, USA). The acquired images were analyzed at $532 \mathrm{~nm}$ for Cy3 detection by Genepix 3.0 software. Differences between the arbitrary two data sets or multiple data sets were tested by Student's $t$ test or one-way ANOVA to each lectin signal using SPSS statistics 20.

\section{Release and analysis of LPS}

The release and analysis of LPS was based on the protocol (Hölzer, Schlumberger, Jäckel, \& Hensel, 2009). Briefly, about $2 \mathrm{~mL}$ A. segnis at OD600 of 0.8-1.2 was washed three times with PBS, centrifuged and resuspended in lysis buffer and boiled at $100^{\circ} \mathrm{C}$ for $10 \mathrm{~min}$. The final concentration of $20 \mathrm{U} / \mathrm{mL}$ DNase I (Yeasen Biotechnology, China) was added to the chilled samples at $37^{\circ} \mathrm{C}$ for $1 \mathrm{~h}$. Then the samples were treated with proteinase $\mathrm{K}$ (Solarbio, China) at $70^{\circ} \mathrm{C}$ for $2 \mathrm{~h}$ according to the manufacturer's recommendation. LPS preparations were separated in $12 \%$ SDS-PAGE and visualized by silver staining using the procedure described by Fomsgaard (Fomsgaard, Freudenberg, \& Galanos, 1990).

\section{Western Blotting}

Protein was extracted from cells using the RIPA buffer (Applygen, China). And $30 \mu \mathrm{g}$ of the protein per lane was separated in 10\% SDS-PAGE gels, and transferred to $0.45 \mu \mathrm{m}$ PVDF membranes (Millipore, USA). Membranes were blocked with $5 \%$ non-fat milk at room temperature for $1 \mathrm{~h}$ and then incubated overnight at $4^{\circ} \mathrm{C}$, with the following primary antibodies: JNK (Proteintech, China), p-JNK (Cell Signaling Technology, USA), p65 (Immunoway, China), p-p65 (Cell Signaling Technology, USA), IL-18 (Proteintech, China), IL-1 $\beta$ (Proteintech, China) and $\beta$-actin (Abways, China). Samples were then incubated with secondary antibodies at $25^{\circ} \mathrm{C}$ for $1 \mathrm{~h}$.

\section{Results}

\section{Overall oral bacterial diversity of $\mathrm{HV}$ and GC cases}

Among the 24 samples, an estimated 79,000 raw sequence reads were obtained for each sample, with a median length of 423 base pairs. After quality trimming and chimera checking, 75 thousand clean 
sequence reads per sample remained, which were recovered for downstream analysis. The detailed sequencing data of each sample was shown in Table S3. In total, 789 unique species were identified in the saliva of HVs and GC patients; 525 species were common in the HVs and GC patients, while 128 and 136 species were observed in only the HVs and GC patients, respectively (Fig. 1A). The alterations of microbial communities between HV and GC groups were evaluated. The alpha-diversity analysis showed that there were no significant differences between two groups (Fig. 1B-E), while the beta-diversity analysis showed that the oral microbiota profiles of the two groups were different, and could be distinguished using PCA, PCoA and NMDS plots (Fig. 1F-H).

\section{Significant variation of oral microbial community in GC patients versus matched HV}

To further investigate the composition of oral microbial communities, the variance at each taxonomic level between the HV and GC groups was compared using the Wilcoxon test (Table 1). The results showed no significant difference at the phylum, class and order levels. However, the family of Carnobacteriaceae was decreased in the GC patients compared with that of HVs (Fig. 2A). The genus of Alloprevotella and Megasphaera were significantly increased whereas genus of Granulicatella, Bregeyella and TM7[G-6] were decreased in the GC group (Fig. 2B). And five species (Oral taxon 392, Oral taxon 308, Aggregatibacter segnis (A. segnis), Megasphaera micronuciformis (M. micronuciformis), and Oral taxon 396) were significantly increased $(p<0.05)$ while two species (Streptococcus salivarius (S. salivarius) and Oral taxon 870 ) were significantly decreased $(p<0.01)$ in GC patients compared with that of HVs (Fig. 2C). Furthermore, PCR and qPCR results showed that the relative abundances of $A$. segnis, $M$.

micronuciformis and S. salivarius were consistent with the results obtained by $16 \mathrm{~S}$ rDNA sequencing (Fig. 2D-E). Further analysis indicated that the combinations of oral bacteria might have the potential as diagnosis biomarkers for GC, although the sample size may not be sufficient (Fig. S2).

Table 1 Taxonomic differences in the oral microbiota of HV and GC. 


\begin{tabular}{lllll}
\multicolumn{2}{c}{ HV } & \multicolumn{2}{c}{ GC } \\
\hline $\begin{array}{l}\text { Avg, } \\
\text { (Carriage, }\end{array}$ & SD & $\begin{array}{l}\text { Avg, } \\
\text { (Carriage, } \\
\text { \%) }\end{array}$ & & \%) \\
\end{tabular}

\section{Phylum _ Bacteroidetes}

C_Bacteroidia; $\quad$ o_Bacteroidales; $\quad$ f_Prevotellaceae;

$0.84(100)$

0.81

$1.85(100)$

1.28

0.032

g_Alloprevotella

C_Bacteroidia; o_Bacteroidales;

g_Alloprevotella; s_sp._oral_taxon_308

C_Bacteroidia; o_Bacteroidales;

f_Prevotellaceae;

$0.27(100)$

0.16

$0.66(100)$

$0.48 \quad 0.019$

f_Prevotellaceae;

$0.05(92)$

0.04

$0.12(100)$

0.110 .046

g_Prevotella; s_sp._oral_taxon_396

C_Flavobacteriia; o_Flavobacteriales; f_Flavobacteriaceae;

$0.42(100)$

0.47

$0.11(100)$

$0.10 \quad 0.047$

g_Bergeyella

Phylum _Firmicutes;

C_Bacilli; o_Lactobacillales; f_Carnobacteriaceae

$2.89(100)$

2.24

1.39 (100)

$0.85 \quad 0.048$

c_Bacilli; o_Lactobacillales; $\quad$ f_Carnobacteriaceae;

2.89 (100)

2.24

1.39 (100)

0.85

0.048

g_Granulicatella

c_Negativicutes; o_Selenomonadales; $f$ _Veillonellaceae;

$0.03(75)$

0.04

$0.15(100)$

$0.17 \quad 0.033$

g_Megasphaera

C_Bacilli; o_Lactobacillales; $\quad$ f_Streptococcaceae;

g_Streptococcus; s_salivarius

$0.27(100)$

0.21

$0.07(100)$

$0.08 \quad 0.007$

c_Negativicutes; o_Selenomonadales; f_Veillonellaceae; g_Megasphaera; s_micronuciformis

$0.03(75)$

0.04

$0.15(100)$

$0.17 \quad 0.036$

Phylum _ Fusobacteria;

C_Fusobacteriia; o_Fusobacteriales; f_Leptotrichiaceae;

g_Leptotrichia; s_sp._oral_taxon_392

$0.46(100)$

0.30

$1.02(100)$

$\begin{array}{ll}0.74 & 0.029\end{array}$

Phylum _ Proteobacteria

c_Gammaproteobacteria;

f_Pasteurellaceae; g_Aggregatibacter; s_segnis

o_Pasteurellales;

$0.10(100)$

0.14

$0.41(75)$

$0.44 \quad 0.032$

Phylum_Saccharibacteria_(TM7)

C_TM7_[C-1]; o_TM7_[O-1]; f_TM7_[F-1]; g_TM7_[G-6]

$0.19(92)$

0.16

$0.03(42)$

$0.05 \quad 0.005$

C_TM7_[C-1]; o_TM7_[O-1]; f_TM7_[F-1]; $\quad g_{-} T M 7 \_[G-6]$;

0.19 (92)

0.16

$0.03(42)$

0.05

0.005 s_sp._oral_taxon_870

\section{Fucose-neoglycoprotein decreased the adhesion of $\boldsymbol{A}$. segnis to oral cells}

Our previous studies have demonstrated that the occurrence and development of GC could induce glycopattern alterations of salivary proteins in the saliva of GC patients, especially fucosylated glycans (Shu et al., 2018). Here, the fucose-neoglycoprotein (Fuc-BSA) and other neoglycoproteins (GalBSA and Man-BSA) were synthesized (Fig. S1) to evaluate their roles in the proliferation and adhesion of A. segnis. The results showed that the proliferation of $A$. segnis was not affected by $3-100 \mu \mathrm{g} / \mathrm{mL}$ FucBSA, Gal-BSA, Man-BSA, monosaccharides (Fuc, Gal, and Man) and BSA (data not shown). However, adding 30 and $100 \mu \mathrm{g} / \mathrm{mL}$ Fuc-BSA significantly decreased the adhesion of $A$. segnis to CAL-27 cells (Fig. $3 A$ ), whereas Man-BSA and Gal-BSA exerted no effects at the same concentration (Fig. S3). Similar phenomenon was observed in HOEC cells, addition of 30 and $100 \mu \mathrm{g} / \mathrm{mL}$ Fuc-BSA also significantly decreased the adhesion of $A$. segnis to HOEC cells (Fig. 3B). In addition, neither BSA nor the free fucose affected the adhesion of $A$. segnis to HOEC cells, indicating that the scaffolding protein is required for the 
function of fucose. These results demonstrated that the fucose moieties of proteins can disturb the attachment of $A$. segnis to the cell surface.

\section{Fuc-BSA reduced the toxicity of $A$. segnis to oral cells}

To investigate the influence of Fuc-BSA on the cytotoxicity of $A$. segnis infection, the viability of HOEC and CAL27 cells was evaluated by CCK8 assay. As shown in Fig. 4, the viability of HOEC and CAL-27 cells was not affected with up to $100 \mu \mathrm{g} / \mathrm{mL}$ Fuc-BSA (Fig. 4A, B). However, when $100 \mu \mathrm{g} / \mathrm{mL}$ Fuc-BSA was added, the toxicity of $A$. segnis ( $\mathrm{MOI} 1 \otimes 5 \otimes 10 \otimes 20$ and 50 ) towards HOEC cells was elevated, with the IC50 increased from $10.67 \mathrm{MOI}$ to $16.4 \mathrm{MOI}$ (Fig. 4C-E). Similar effect of Fuc-BSA was also observed with CAL27 cells, with the IC50 increased from $9.72 \mathrm{MOI}$ to $12.3 \mathrm{MOI}$ ((Fig. 4F-H). These results demonstrated that Fuc-BSA can reduce the toxicity of $A$. segnis to oral cells.

\section{Fuc-BSA changed the glycopatterns of $A$. segnis}

Saliva is one of the most important environment factors in oral-bacteria interactions, and it is unclear whether the altered salivary glycopatterns effect the condition of $A$. segnis. Therefore, $A$. segnis was consecutively subcultured in the medium containing 30 and $100 \mu \mathrm{g} / \mathrm{mL}$ Fuc-BSA on each day up to 15 days to ensure the cells were exposed to enough Fuc-BSA stress and develop any adaptive alteration. Then the glycopatterns of outer membrane and total proteins of $A$. segnis were analyzed by lectin microarrays. Three lectins (PHA-E, LEL, and MAL-I) showed extremely increased expression levels of the glycopatterns (bisecting GlcNAc, high mannose-type N-glycans, and Gal $\beta-1,4 \mathrm{GlcNAc}$ ), while 9 lectins (e.g., Jacalin, GSL-II, and SNA) showed decreased expression levels of the glycopatterns (e.g., Galß1-3GalNAcaSer/Thr, GalNAca-Ser/Thr and Sia2-6Gal/GalNAc) on the outer membrane of $A$. segnis after Fuc-BSA treatment (Fig. 5A). However, 4 lectins (PHA-E, PNA, LEL and GSL-I) showed increased expression levels of glycopatterns (bisecting GIcNAc, Galß1-3GalNAca-Ser/Thr, high mannose-type N-glycans, and $\mathrm{aGalNAc/aGal)}$ in the total proteins of $A$. segnis treated with Fuc-BSA (Fig. 5B). The glycopattern changes in the outer membrane was more dramatic than that of the total proteins after 15 days co-culture with Fuc-BSA, which implied that Fuc-BSA mainly affected the glycopatterns of outer membrane in $A$. segnis. The sum NFIs of all up-regulated lectins including PHA-E (2.39\% - 3.09\% - 13.23\%), LEL $(0.92 \%-2.51 \%$ $24.9 \%)$ and MAL-I $(2.78 \%-3.31 \%-18.88 \%)$ were increased from $6.09 \%$ to $8.91 \%$ and to $57.17 \%$ at 0,30 , and $100 \mu \mathrm{g} / \mathrm{mL}$ Fuc-BSA treatment, respectively. It is interesting that the glycans recognized by these three lectins are typical $\mathrm{N}$-linked glycans. And it is speculated that the dramatic reduction of O-linked glycans in the outer membrane may be related to the synthesis of LPS.

\section{Fuc-BSA affected the biosynthesis of LPS in A. segnis}

Annotation of $A$. segnis in the carbohydrate-active enzymes database (CAZy) revealed a totally of 24 putative glycosyltransferases (GTs) distributed among 11 GT families. Of these, 8 GTs are associated with LPS biosynthesis, 7 GTs are associated with cell wall formation, and the rest are associated with glycosylation, glycogen synthase, and carbohydrate metabolism (Table S4). The LPS-associated GTs and glycosylation-associated GTs were investigated in this study. As a result, the qPCR showed that three 
mRNAs of LPS-associated GTs (NCTC10977_00605 (Rfaq_1), NCTC10977_00729, and NCTC10977_00649 (WaaA)) were significantly decreased and the mRNA of mannosyltransferase OCH1 was significantly increased in A. segnis after Fuc-BSA treatment (Fig. 5C), which suggested that Fuc-BSA might interfered with the biosynthesis of core oligosaccharides or O-antigenic polysaccharides of LPS. SDS-PAGE and silver staining showed that the length of LPS has changed significantly. The truncated LPS appeared in the Fuc-BSA treated $A$. segnis suggested that the synthesis pathway of LPS was disrupted (Fig. 5D), which might be responsible for the changed outer membrane glycopatterns in $A$. segnis after Fuc-BSA treatment.

\section{Fuc-BSA enhanced the capacity of A. segnis to trigger innate immune response}

Transcription and expression of IL-1 $\beta$, IL-18 and IFN- $\beta$ are important markers and mediators of LPSinduced innate immunity. Here, QPCR and western blotting were used to evaluate the innate immunity activation by $A$. segnis and Fuc-BSA treated $A$. segnis in RAW264.7 cells. The qPCR results showed that mRNA levels of IL-1 $\beta$, IL-18 and IFN- $\beta$ were significantly increased after $24 \mathrm{~h}$ of $5 \mathrm{MOI} A$. segnis infection. However, the transcriptional levels of IL-1 $\beta$, IL-18 and IFN- $\beta$ elevated even higher when infected with $A$. segnis ( $5 \mathrm{MOI}$ ) treated by Fuc-BSA ( 30 and $100 \mu \mathrm{g} / \mathrm{mL}$ ) (Fig. 6A-C). Western blotting analysis was used to verify these changes of IL-18 and IL-1 $\beta$, and showed that in comparison with control group, exposure to $A$. segnis and Fuc-BSA treated $A$. segnis in RAW264.7 cells elevated the levels of phosphorylated p65 and JNK, which were the key factors in the NF-KB and JNK signaling pathways (Fig. 6D, E). These results demonstrated that Fuc-BSA enhanced the capacity of $A$. segnis to trigger innate immune responses in RAW264.7 cells.

\section{Discussion}

Some risk factors, such as $\mathrm{H}$. pylori infection, cigarette smoking, alcohol drinking, salted preserved food intake, have previously been confirmed to contribute to GC development, but still a large number of new cases are diagnosed each year that are not associated with any of these plausible etiologic explanations. Hence, it remains a critical requirement to investigate the effective biomarkers and definite molecular mechanism of GC. With the implementation and progress of human microbiology project, the important role of human microbiome in human healthy has emerged as the focal point, and the importance of intestinal microbiota to human health has been widely studied (Y. Fan \& Pedersen, 2021; Lee et al., 2021). As another major habitat, oral cavity is inhabited by various microorganisms that usually exist in a balanced immuno-inflammatory state with the host (Whitmore \& Lamont, 2014). Several epidemiological studies have observed a strong risk association between periodontal disease and GC (Michaud, Fu, Shi, \& Chung, 2017; Michaud, Kelsey, Papathanasiou, Genco, \& Giovannucci, 2016; Ndegwa et al., 2018). However, the causal relationship between periodontal disease and GC are still disputed, and the underlying mechanism between oral microbiota and GC has not yet been clarified. Hence, exploring the difference in oral microbiota between HV and GC patients could help understand the role of oral microbiota in GC development. 
In this study, the oral microbiota of GC patients was first investigated by 16S rDNA high-throughput sequencing. Visible differences were observed at species level in GC compared to HV cases. And the results indicated that the combinations of oral bacteria might have the potential to act as diagnostic biomarkers for GC. Among these seven altered bacteria, $A$. segnis and M. micronuciformis could be cultivated and showed increased abundance in the GC patients. The M. micronuciformis was not preserved in all the culture collections, nor was it separated in this study. Haemophilus segnis was first isolated from dental plaque in 1977 (Kilian, 1976) and reclassified into the Aggregatibactergenus in 2006 (Nørskov-Lauritsen \& Kilian, 2006). It is a gram-negative bacteria, a normal commensal in human oral cavity, but rarely reported as a pathogen. Infective endocarditis, periodontal disease, acute appendicitis, pancreatic abscess, and cholecystitis was reported to be associated with A. segnis (Carson, Rezmer, \& Belli, 1997; Revest, Egmann, Cattoir, \& Tattevin, 2016; Welch, Southern, \& Schneider, 1986). Previous studies about $A$. segnis mainly focused on case reports, the true prevalence of this bacterium in human infections is probably underreported, and the underlying mechanism associated with diseases is not yet explicitly known.

The glycoprotein components of saliva play a pivotal role in modulating the oral microbiota. Particular components of saliva, such as salivary mucins (e.g. MUC5B , MUC7, MUC19) and salivary agglutinins (agglutinin gp340/DMBT1, secretory IgA, free secretory component), participate in the clearance of oral bacteria (Cross \& Ruhl, 2018; Frenkel \& Ribbeck, 2015; Xu et al., 2017). Our studies demonstrated that human disease could induce different alterations of salivary protein glycopatterns (Liu et al., 2018; Zhang et al., 2019). For example, the occurrence and development of GC could induce alterations in salivary protein glycopatterns, especially fucosylated glycopattern in the saliva of GC patients (Shu et al., 2018). And it has never been studied whether and how the salivary glycopatterns variation affect the oral microbiota. Therefore, three neoglycoproteins were synthesized corresponding to the altered salivary glycopatterns in GC patients to simulate and explore the effects of glycan for $A$. segnis. The results revealed that only Fuc-BSA could significantly attenuate the adhesion ability of $A$. segnis to HOEC and CAL-27 cells, while other neoglycoproteins (Man-BSA and Gal-BSA) and three free monosaccharides (Fuc, Man and Gal) did not have the same effect. Besides, Fuc-BSA could also reduce the toxicity of $A$. segnis to oral cells, which might be related to the number of attached bacteria decreased. And the more intriguing observation was that constant stimulations of Fuc-BSA to $A$. segnis changed glycan structures on the surface of $A$. segnis, especially in the LPS of the A. segnis. LPS is composed of lipid A, core oligosaccharide and $\mathrm{O}$-antigenic polysaccharide (O-antigen). The lipid $\mathrm{A}$ is a highly conserved lipid moiety and indispensable for bacterial survival and growth. The 0 -antigen is an important component to against host immunity. Some pathogens, such as Burkholderia cepacia, Salmonella enterica and Francisella tularensis, depend on 0-antigen to avoid phagocytosis and escape the lytic action of host cells (Kalynych, Morona, \& Cygler, 2014). The long-0-antigen can inhibit the binding of LPS to CD14, thus affecting the activation of TLR4-TRIF and reducing the host's immune response against Leptospirosis and contribute to escape the innate immune defenses, whereas the truncated induces more TLR4-TRIF responses (Bonhomme et al., 2020). The synthesis of integrated LPS was disrupted, resulting in the 
display of truncated LPS on the surface of $A$. segnis. This might be responsible for the stronger innate immune responses induced by Fuc-BSA treated $A$. segnis in RAW264.7 cells.

In conclusion, the present study investigated the differences of the oral microbiome between HVs and GC patients. There were five species that were significantly increased while two species were significantly decreased $(p<0.01)$ in the saliva of GC patients compared with that of HVs. The fucose-neoglycoproteins could reduce the adhesion and toxicity of $A$. segnis to oral cells, change the glycan structures in the LPS of $A$. segnis, and enhance the capacity of $A$. segnis via the truncated LPS to trigger innate immune responses in RAW264.7 cells (Fig.7A). It hypothesized that decreased expression of fucosylated glycopattterns in the GC patients improves the adhesion of $A$. segnis and enhance the immune escape via the long-O-antigen of LPS, due to the high abundance $A$. segnis in oral cavity (Fig.7B). These findings provided important clues that the dysbiosis of the oral microbiota might be naturally regulated by salivary protein glycopatterns in GC patients, and had important implications for elucidating the basis of glycanmediated interactions between host cells and native bacteria, as well as having the potential for developing new carbohydrate drugs for oral health.

\section{References}

Belda-Ferre, P., Alcaraz, L. D., Cabrera-Rubio, R., Romero, H., Simón-Soro, A., Pignatelli, M., \& Mira, A. (2012). The oral metagenome in health and disease. Isme j, 6(1), 46-56.

Bonhomme, D., Santecchia, I., Vernel-Pauillac, F., Caroff, M., Germon, P., Murray, G., . . Werts, C. (2020). Leptospiral LPS escapes mouse TLR4 internalization and TRIF-associated antimicrobial responses through 0 antigen and associated lipoproteins. PLoS Pathog, 16(8), e1008639.

Carson, H. J., Rezmer, S., \& Belli, J. (1997). Haemophilus segnis cholecystitis: a case report and literature review. $J$ Infect, 35(1), 85-86.

Cross, B. W., \& Ruhl, S. (2018). Glycan recognition at the saliva - oral microbiome interface. Cell Immunol, 333, 19-33.

Dewhirst, F. E., Chen, T., Izard, J., Paster, B. J., Tanner, A. C., Yu, W. H., . . Wade, W. G. (2010). The human oral microbiome. J Bacteriol, 192(19), 5002-5017.

Escapa, I. F., Chen, T., Huang, Y., Gajare, P., Dewhirst, F. E., \& Lemon, K. P. (2018). New Insights into Human Nostril Microbiome from the Expanded Human Oral Microbiome Database (eHOMD): a Resource for the Microbiome of the Human Aerodigestive Tract. mSystems, 3(6), e00187-00118.

Fan, X., Alekseyenko, A. V., Wu, J., Peters, B. A., Jacobs, E. J., Gapstur, S. M., . . Ahn, J. (2018). Human oral microbiome and prospective risk for pancreatic cancer: a population-based nested case-control study. Gut, 67(1), 120-127. 
Fan, Y., \& Pedersen, O. (2021). Gut microbiota in human metabolic health and disease. Nat Rev Microbiol, 19(1), 55-71.

Farrell, J. J., Zhang, L., Zhou, H., Chia, D., Elashoff, D., Akin, D., . . Wong, D. T. (2012). Variations of oral microbiota are associated with pancreatic diseases including pancreatic cancer. Gut, 61(4), 582-588.

Flemer, B., Warren, R. D., Barrett, M. P., Cisek, K., Das, A., Jeffery, I. B., .. O'Toole, P. W. (2018). The oral microbiota in colorectal cancer is distinctive and predictive. Gut, 67(8), 1454-1463.

Fomsgaard, A., Freudenberg, M. A., \& Galanos, C. (1990). Modification of the silver staining technique to detect lipopolysaccharide in polyacrylamide gels. J Clin Microbiol, 28(12), 2627-2631.

Frenkel, E. S., \& Ribbeck, K. (2015). Salivary mucins in host defense and disease prevention. J Oral Microbiol, 7, 29759.

Hölzer, S. U., Schlumberger, M. C., Jäckel, D., \& Hensel, M. (2009). Effect of the O-antigen length of lipopolysaccharide on the functions of Type III secretion systems in Salmonella enterica. Infect Immun, 77(12), 5458-5470.

Irfan, M., Delgado, R. Z. R., \& Frias-Lopez, J. (2020). The Oral Microbiome and Cancer. Front Immunol, 11, 591088.

Kalynych, S., Morona, R., \& Cygler, M. (2014). Progress in understanding the assembly process of bacterial 0-antigen. FEMS Microbiol Rev, 38(5), 1048-1065.

Kawakubo, M., Ito, Y., Okimura, Y., Kobayashi, M., Sakura, K., Kasama, S., . . Nakayama, J. (2004). Natural antibiotic function of a human gastric mucin against Helicobacter pylori infection. Science, 305(5686), 1003-1006.

Kilian, M. (1976). A taxonomic study of the genus Haemophilus, with the proposal of a new species. $J$ Gen Microbiol, 93(1), 9-62.

Lamont, R. A.-O., Koo, H., \& Hajishengallis, G. A.-O. (2018). The oral microbiota: dynamic communities and host interactions. Nat Rev Microbiol, 16(12), 745-759.

Lee, K. A., Luong, M. K., Shaw, H., Nathan, P., Bataille, V., \& Spector, T. D. (2021). The gut microbiome: what the oncologist ought to know. Br J Cancer.

Ling, Z., Liu, X., Cheng, Y., Jiang, X., Jiang, H., Wang, Y., \& Li, L. (2015). Decreased Diversity of the Oral Microbiota of Patients with Hepatitis B Virus-Induced Chronic Liver Disease: A Pilot Project. Sci Rep, 5 , 17098.

Liu, X., Yu, H., Qiao, Y., Yang, J., Shu, J., Zhang, J., . . Li, Z. (2018). Salivary Glycopatterns as Potential Biomarkers for Screening of Early-Stage Breast Cancer. EBioMedicine, 70-79. 
Long, J., Cai, Q., Steinwandel, M., Hargreaves, M. K., Bordenstein, S. R., Blot, W. J., . . Shu, X. O. (2017). Association of oral microbiome with type 2 diabetes risk. J Periodontal Res, 52(3), 636-643.

Lynge Pedersen, A. M., \& Belstrøm, D. (2019). The role of natural salivary defences in maintaining a healthy oral microbiota. J Dent, 80 Suppl 1, S3-s12.

Meurman, J. H. (2010). Oral microbiota and cancer. J Oral Microbiol, 2.

Michaud, D. S., Fu, Z., Shi, J., \& Chung, M. (2017). Periodontal Disease, Tooth Loss, and Cancer Risk. Epidemiol Rev, 39(1), 49-58.

Michaud, D. S., Kelsey, K. T., Papathanasiou, E., Genco, C. A., \& Giovannucci, E. (2016). Periodontal disease and risk of all cancers among male never smokers: an updated analysis of the Health Professionals Follow-up Study. Ann Oncol, 27(5), 941-947.

Ndegwa, N., Ploner, A., Liu, Z., Roosaar, A., Axéll, T., \& Ye, W. (2018). Association between poor oral health and gastric cancer: A prospective cohort study. Int J Cancer, 143(9), 2281-2288.

Nørskov-Lauritsen, N., \& Kilian, M. (2006). Reclassification of Actinobacillus actinomycetemcomitans, Haemophilus aphrophilus, Haemophilus paraphrophilus and Haemophilus segnis as Aggregatibacter actinomycetemcomitans gen. nov., comb. nov., Aggregatibacter aphrophilus comb. nov. and Aggregatibacter segnis comb. nov., and emended description of Aggregatibacter aphrophilus to include $V$ factor-dependent and V factor-independent isolates. Int J Syst Evol Microbiol, 56(Pt 9), 2135-2146.

Peters, B. A., Wu, J., Pei, Z., Yang, L., Purdue, M. P., Freedman, N. D., . . Ahn, J. (2017). Oral Microbiome Composition Reflects Prospective Risk for Esophageal Cancers. Cancer Res, 77(23), 6777-6787.

Revest, M., Egmann, G., Cattoir, V., \& Tattevin, P. (2016). HACEK endocarditis: state-of-the-art. Expert Rev Anti Infect Ther, 14(5), 523-530.

Shang, F. M., \& Liu, H. L. (2018). Fusobacterium nucleatum and colorectal cancer: A review. World J Gastrointest Oncol, 10(3), 71-81.

Shu, J., Ma, J., Ren, X., Wang, J., Wang, Y., Zhang, K., .. Li, Z. (2021). The Abnormal Glycopatterns of Salivary Glycoproteins in Esophageal Squamous Cell Carcinoma Patients Front Chem, 9, 637730.

Shu, J., Yu, H., Du, H., Zhang, J., Zhang, K., Li, X., . . Li, Z. (2018). Identification of N- and O-linked glycans recognized by $A A L$ in saliva of patients with atrophic gastritis and gastric cancer. Cancer Biomarkers, 22(4), 669-681.

Shu, J., Yu, H., Li, X., Zhang, D., \& Liu, X. (2017). Salivary glycopatterns as potential biomarkers for diagnosis of gastric cancer. oncotarget, 8(22), 35718-35727. 
Sun, J., Tang, Q., Yu, S., Xie, M., Xie, Y., Chen, G., \& Chen, L. (2020). Role of the oral microbiota in cancer evolution and progression. Cancer Med, 9(17), 6306-6321.

Sun, J., Zhou, M., Salazar, C. R., Hays, R., Bedi, S., Chen, Y., \& Li, Y. (2017). Chronic Periodontal Disease, Periodontal Pathogen Colonization, and Increased Risk of Precancerous Gastric Lesions. J Periodontol, 88(11), 1124-1134.

Welch, W. D., Southern, P. M., Jr., \& Schneider, N. R. (1986). Five cases of Haemophilus segnis appendicitis. J Clin Microbiol, 24(5), 851-852.

Whitmore, S. E., \& Lamont, R. J. (2014). Oral bacteria and cancer. PLoS Pathog, 10(3), e1003933.

Wu, J., Xu, S., Xiang, C., Cao, Q., Li, Q., Huang, J., . . Zhan, Z. (2018). Tongue Coating Microbiota Community and Risk Effect on Gastric Cancer. J Cancer, 9(21), 4039-4048.

Xu, D., Pavlidis, P., Taskent, R. O., Alachiotis, N., Flanagan, C., DeGiorgio, M., . . Gokcumen, O. (2017). Archaic Hominin Introgression in Africa Contributes to Functional Salivary MUC7 Genetic Variation. Mol Biol Evol, 34(10), 2704-2715.

Yang, J., Zhou, Y., Zhang, L., Shah, N., Jin, C., Palmer, R. J., Jr., \& Cisar, J. O. (2016). Cell Surface Glycoside Hydrolases of Streptococcus gordonii Promote Growth in Saliva. Appl Environ Microbiol, 82(17), 52785286.

Zhang, J., Zhong, Y., Zhang, P., Du, H., Shu, J., Liu, X., . . Li, Z. (2019). Identification of abnormal fucosylated-glycans recognized by LTL in saliva of HBV-induced chronic hepatitis, cirrhosis, and hepatocellular carcinoma. Glycobiology, 29(3), 242-259.

\section{Declarations}

Funding: This study was supported by the National Natural Science Foundation of China (Grant No. 32101030), the China Postdoctoral Science Foundation (Grant No. 2020M673628XB), and the Natural Science Foundation of Shaanxi Province (Grant No. 2021JQ-446).

Author contributions: JS, HY, ZL, BL designed the experiments; JS, XR, YW, KZ, ZT performed experiments and analyzed the data; JS, XR, WC, LD and ZL wrote the paper.

Competing interests: We declare no competing interests.

\section{Figures}


A

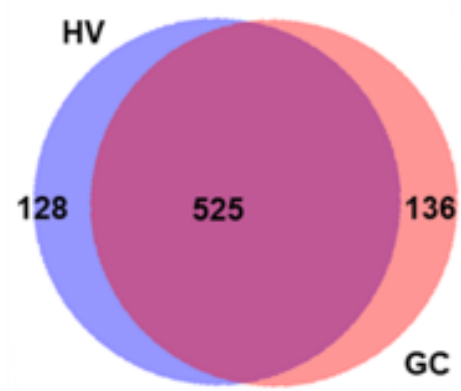

Venn diagram
B

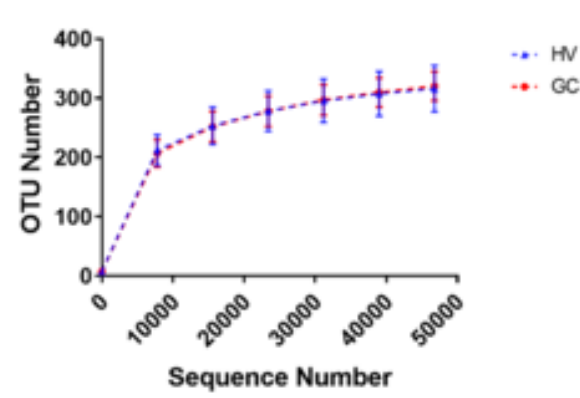

D

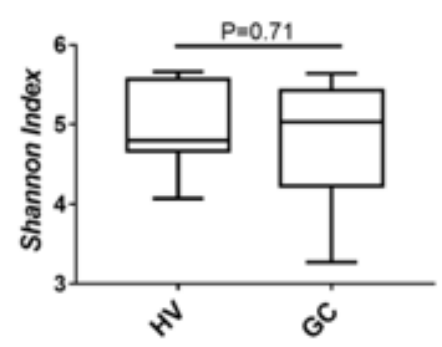

G

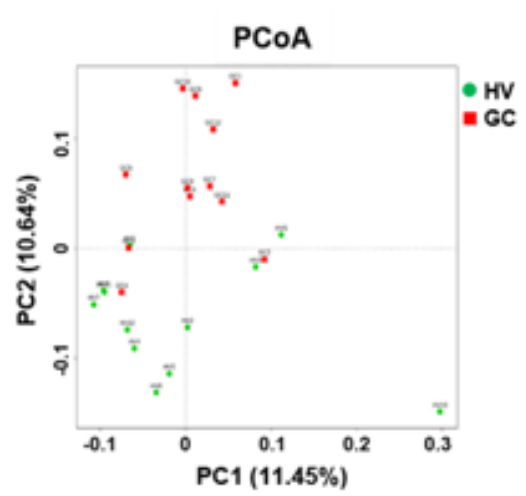

C

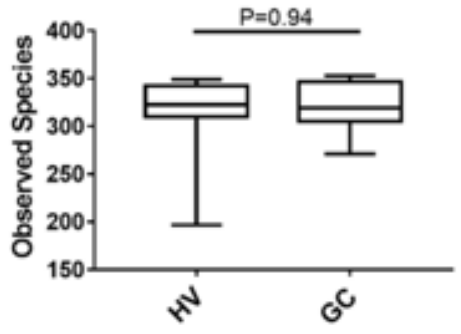

E
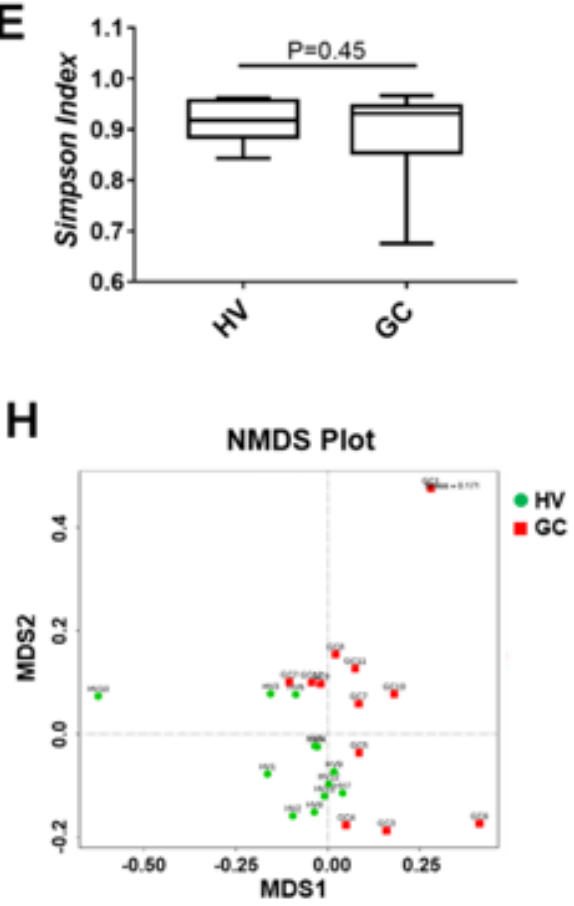

Figure 1

Comparison of oral microbiota structures between the HV and GC groups. (A) Venn diagram illustrated the overlap of the species identified in the oral microbiota of HV and GC groups, (B) OTU numbers, (C) Observed species, (D) Shannon index, (E) Simpson index, (F) PCA, (G) PCoA, (H) NMDS analysis.

Figure 2

Differences in the oral microbiota between HV and GC groups. Comparison of the relative abundance at the bacterial family (A), genus (B) and species (C) levels in the HV and GC groups. (D) Agarose gel electrophoresis of the PCR products and the box plot of the relative intensities. (E) The qPCR results of $A$. segnis, M. micronuciformis and S. salivarius.

Figure 3 
The adhesion of A. segnis to CAL-27 (A) and HOEC (B) cells. A. segnis was added to cells with Fuc-BSA and allowed to infect for $90 \mathrm{~min}$. Fuc-BSA (30 and $100 \mu \mathrm{g} / \mathrm{mL}$ ) significantly reduced the adhesion of $A$. segnis to CAL-27 and HOEC cells. The images are acquired under the same conditions for the merged channels of FITC $(\operatorname{Ex} / \operatorname{Em}(n m)=488 / 520)$, DiD $(\operatorname{Ex} / \operatorname{Em}(n m)=650 / 670)$ and DAPI $(\operatorname{Ex} / \operatorname{Em}(n m)=358 / 461)$. Statistical significance analysis for FITC fluorescence intensity per cell was computed by one-way ANOVA $(*: p<0.05, * *: p<0.01$, and $* * *: p<0.001)$.
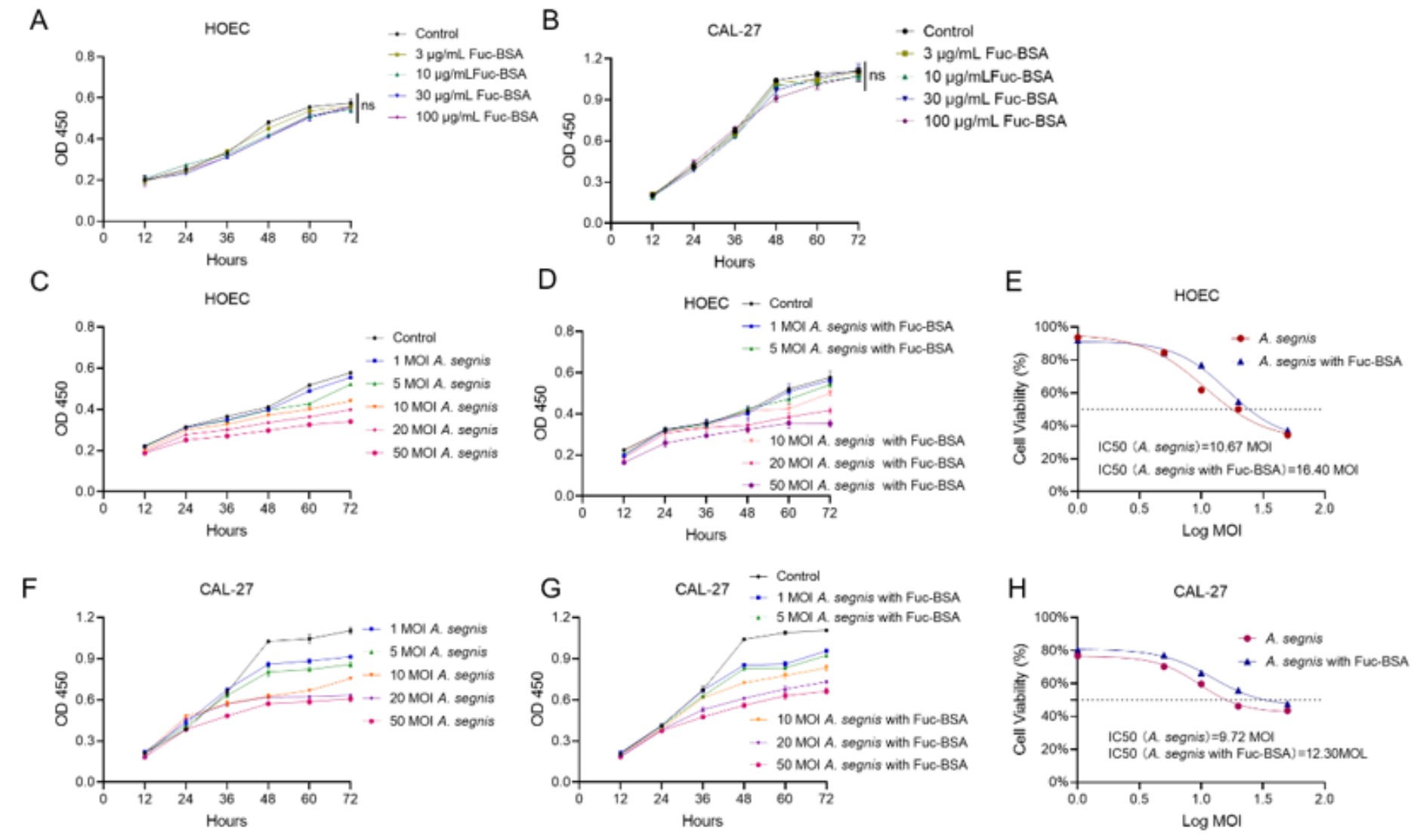

\section{Figure 4}

The effect of Fuc-BSA on the toxicity of A. segnis to oral cells. Cell viabilities of HOEC (A) and CAL-27 cells (B) treated with Fuc-BSA at different concentrations. Cell viabilities of HOEC (C) and CAL-27 cells (D) treated with $A$. segnis (MOI $1 \varangle 5 \rrbracket 10 \rrbracket 20$ and 50$)$. Cell viability curve of HOEC (E) and CAL-27 cells $(H)$ treated with A. segnis were accompanied and not accompanied by $100 \mu \mathrm{g} / \mathrm{mL}$ Fuc-BSA. Cell viabilities of HOEC (F) and CAL-27 cells (G) treated with A. segnis was accompanied by $100 \mu \mathrm{g} / \mathrm{mL}$ Fuc-BSA. Fuc-BSA did not affect the viability of HOEC and CAL-27 cells, and addition of Fuc-BSA significantly reduced the toxicity of A. segnis to HOEC and CAL-27 cells.

\section{Figure 5}

The glycopatterns of A. segnis were altered by Fuc-BSA treatment. (A) The outer membrane glycopatterns analyzed by lectin microarrays. Nine lectins showed decreased NFIs in the detection of outer membrane 
glycopatterns after Fuc-BSA treatment ( 30 and $100 \mu \mathrm{g} / \mathrm{mL}$ ), while three lectins showed extremely increased NFIs. (B) The total protein glycopatterns analyzed by lectin microarrays. Four lectins showed increased NFIs in the detection of total protein glycopatterns after Fuc-BSA treatment (30 and 100 $\mu \mathrm{g} / \mathrm{mL}$ ). (C) The RT-qPCR results of glycosyltransferases in A. segnis. The mRNA levels of three LPSrelated glycosyltransferases (NCTC10977_00605, 00729 and 0069) decreased and another glycosyltransferase (mannosyltransferase $\mathrm{OCH} 1$ ) increased in A. segnis after Fuc-BSA treatment. (D) The SDS-PAGE and silver staining analysis of LPS released from A. segnis. The LPS from Fuc-BSA treated A. segnis was altered compared with untreated A. segnis. E. coli: $10 \mu \mathrm{g}$ LPS from E. coli 055:B5, 1: the LPS from A. segnis, 2: the LPS from A. segnis treatment with $30 \mu \mathrm{g} / \mathrm{mL}$ Fuc-BSA, 3: the LPS from A. segnis treatment with $100 \mu \mathrm{g} / \mathrm{mL}$ Fuc-BSA, M: Pre-stained protein marker.

\section{Figure 6}

Treatment with Fuc-BSA enhanced the capacity of A. segnis to trigger innate immune responses in Raw 264.7 cells. The RT-qPCR results of IL-1 $\beta$ (A)IL-18 (B) and IFN- $\beta$ (C) in RAW264.7 cells incorporated with A. segnis and Fuc-BSA treated A. segnis, respectively. Fuc-BSA (30 and $100 \mu \mathrm{g} / \mathrm{mL}$ ) treated A. segnis (5 $\mathrm{MOI}$ ) elevated higher mRNAs level of IL-1 $\beta \square \mathrm{IL}-18$ and IFN- $\beta$ compared with $A$. segnis. (D) Western blotting analysis of IL-1 $\beta$ and IL-18 expression in RAW264.7 cells incorporated with A. segnis and Fuc-BSA treated A. segnis, respectively. (E) Western blotting detection of p-p65 and p-JNK expression in RAW264.7 cells incorporated with A. segnis and Fuc-BSA treated A. segnis, respectively. Fuc-BSA treatment A. segnis elevated the levels of phosphorylated p65 and JNK in RAW264.7 cell. Fuc-BSA treated A. segnis triggered stronger innate immune responses in RAW264.7 cells.

\section{Figure 7}

The effect of Fuc-BSA to A. segnis and the hypothesis of the effect of altered salivary glycopatterns to oral microbiota in GC patients. (A) The adhesion and cytotoxicity of A. segnis to oral cells were reduced by $30-100 \mu \mathrm{g} / \mathrm{mL}$ Fuc-BSA. And the capacity of A. segnis to trigger innate immune responses in RAW264.7 was enhanced by constant stimulation of Fuc-BSA. (B) Decreased expression of fucosylated glycans in the GC patients improves the adhesion of $A$. segnis and enhance the immune escape via the long-O-antigen of LPS, due to the high abundance A. segnis in oral cavity.

\section{Supplementary Files}

This is a list of supplementary files associated with this preprint. Click to download.

- SupplementaryMaterials.docx 